\title{
Cortical Volumetric Correlates of Childhood Trauma, Anxiety, and Impulsivity in Bipolar Disorder
}

\author{
Hyehyun Song1, Myong-Wuk Chon', Vin Ryu², Rina Yu², Dong-Kyun Lee², \\ Hyeongrae Lee ${ }^{2}$, Wonhye Lee ${ }^{3}$, Jung Hyun Lee ${ }^{1}$, and Dong Yeon Park ${ }^{4} \bowtie$ \\ ${ }^{1}$ Department of Psychiatry, National Center for Mental Health, Seoul, Republic of Korea \\ 2Department of Mental Health Research, National Center for Mental Health, Seoul, Republic of Korea \\ ${ }^{3}$ Department of Clinical Psychology, National Center for Mental Health, Seoul, Republic of Korea \\ ${ }^{4}$ Department of Mood Disorders, National Center for Mental Health, Seoul, Republic of Korea
}

Objective More recently, attention has turned to the linkage between childhood trauma and emotional dysregulation, but the evidence in bipolar disorder (BD) is limited. To determine neurobiological relationships between childhood trauma, current anxiety, and impulsivity, we investigated cortical volumetric correlates of these clinical factors in BD.

Methods We studied 36 patients with DSM-5 BD and 29 healthy controls. Childhood trauma, coexisting anxiety, and impulsivity were evaluated with the Korean version-Childhood Trauma Questionnaire (CTQ), the Korean version-Beck Anxiety Inventory (BAI), and the Korean version-Barratt Impulsiveness Scale (BIS). Voxel-based morphometry (VBM) was used to assess gray matter volume (GMV) alterations on the brain magnetic resonance imaging (MRI). Partial correlation analyses were conducted to examine associations between the GMV and each scale in the BD group.

Results Childhood trauma, anxiety, and impulsivity were interrelated in BD. BD patients revealed significant inverse correlations between the GMV in the right precentral gyrus and CTQ scores $(r=-0.609, p<0.0003)$; between the GMV in the left middle frontal gyrus and BAI scores $(r=-0.363, p=0.044)$. Moreover, patients showed similar tendency of negative correlations between the GMV in the right precentral gyrus and BIS scores; between the GMV in the left middle frontal gyrus and CTQ scores.

Conclusion The present study provides evidence for a neural basis between childhood trauma and affect regulations in BD. The GMV alterations in multiple frontal lobe areas may represent neurobiological markers for anticipating the course of BD.

Psychiatry Investig 2020;17(7):627-635

Key Words Bipolar disorder, Gray matter volume, Childhood trauma, Anxiety, Impulsivity.

\section{INTRODUCTION}

Bipolar disorder (BD) is a chronic mental illness entailing recurrent mood episodes. ${ }^{1}$ It has been well documented that $\mathrm{BD}$ is associated with high morbidity, disability, and risk of suicide. ${ }^{2}$ A number of studies have explored risk indicators of unfavorable outcomes in $\mathrm{BD}$, and several emerging literatures indicate that adverse childhood experiences, comorbid anxiety, and impulsivity often complicate the presentation

\section{Received: November 13, 2019 Revised: February 20, 2020} Accepted: March 17, 2020

$\triangle$ Correspondence: Dong Yeon Park, MD, PhD

Department of Mood Disorders, National Center for Mental Health, 127 Yongmasan-ro, Gwangjin-gu, Seoul 04933, Republic of Korea

Tel: +82-2-2204-0113, Fax: +82-2-2204-0390, E-mail: dongyeonp@gmail.com

(a) This is an Open Access article distributed under the terms of the Creative Commons Attribution Non-Commercial License (https://creativecommons.org/licenses/by$\mathrm{nc} / 4.0$ ) which permits unrestricted non-commercial use, distribution, and reproduction in any medium, provided the original work is properly cited. and the course of BD. ${ }^{3-5}$

$\mathrm{BD}$ is the psychiatric diagnosis most often associated with a history of childhood trauma. ${ }^{6}$ Bipolar patients who experienced maltreatment in their early lives are prone to have an earlier onset, a worse clinical course, and higher suicidality., Besides, $\mathrm{BD}$ patients with lifetime anxiety disorders appear to have a negative course, including higher numbers of mood episodes, more severe depressive and mixed episodes, and higher levels of suicidality. ${ }^{9-12}$ Furthermore, coexisting impulsivity in BD seems to be a crucial determinant of clinical outcomes. ${ }^{13,14}$ The severity of suicidal attempts in BD has been related to the level of impulsivity. ${ }^{15,16}$

More recently, attention has been paid to the linkage among childhood trauma, current anxiety, and impulsivity in BD. A few researches reported that childhood abuse was significantly associated with affective instability in $\mathrm{BD}$ independent of cur- 
rent mood states, ${ }^{17}$ and that $\mathrm{BD}$ patients showed considerably higher impulsivity when anxiety was present either as a comorbidity or a symptom. ${ }^{18}$ One possible mechanism underlying these observations is the disruption of key stress-response systems during early stages of child development, such as the hypothalamic-pituitary-adrenal axis, serotonin and catecholamine systems, and neurotrophic factors. These disturbances can substantially influence stress handling, arousal, and emotional behavior, leading to an increased allostatic load and negative consequences in brain development in the long term. ${ }^{19}$ Despite the critical role of the childhood traumas and comorbid affective instability in $\mathrm{BD}$, less is known about their relationships and neuroanatomical correlates.

The results of neuroimaging studies in BD have been inconsistent due to complicating factors. Reasons for the discrepancies include small sample sizes, heterogeneity of subject samples, and differences in methods between studies such as imaging acquisition, processing, and analyses..$^{20}$ Nonetheless, neuroimaging researches using voxel-based morphometry (VBM), a computational method to analyze gray matter volume (GMV) on the brain magnetic resonance imaging (MRI) scans, have indicated the involvement of cortical or subcortical structures in the pathophysiology of BD. ${ }^{20,21}$ These findings would contribute to a better understanding of neurobiological mechanisms of $\mathrm{BD}$, and provide evidence for future hypotheses and models about the roles of critical clinical factors in the disorder.

To date, there is a scarcity of neuroimaging studies on the role of childhood trauma, comorbid anxiety, and impulsivity in BD. Bipolar patients with adverse childhood experiences compared to healthy individuals exhibited GMV loss in the bilateral orbitofrontal cortex and the thalamus.2 Similarly, GMV in the right dorsolateral prefrontal cortex and the right thalamus was smaller in BD patients with childhood traumas than patients without them. ${ }^{19}$ In familial pediatric $\mathrm{BD}$, there was a significant negative association between the total hippocampal volume and anxiety scores. ${ }^{20}$ To our knowledge, no research exists integrating the structural brain correlates of various and comprehensive clinical aspects of BD.

The present study aimed to examine cortical or subcortical volumetric correlates of the three paramount clinical variables described above in bipolar patients. First, childhood trauma, comorbid anxiety, and impulsivity of BD patients were assessed via standardized clinical scales, and the relationships between each scale were explored. Second, the correlations between GMV alterations and the scores of the clinical scales were analyzed within the BD group. We hypothesized that GMV changes in the brain would be associated with childhood trauma, anxiety, and impulsivity in $\mathrm{BD}$, and that the brain regions with such alterations would overlap between the patients.

\section{METHODS}

\section{Subjects}

36 patients with BD I or II and 29 healthy individuals between 18 and 49 years were recruited from the Department of Mood Disorder at the National Center for Mental Health, Seoul, Republic of Korea, from 2017 to 2018. Patients were composed of 27 inpatients and nine outpatients at the time of enrollment. All subjects were interviewed by a psychiatrist who used the Korean version of the Mini-International Neuropsychiatric Interview (MINI). ${ }^{23,24}$ Best-estimate lifetime diagnoses were made according to the Diagnostic and statistical manual of mental disorders (DSM-5) $)^{25}$ criteria. In cases with a doubt, a diagnosis was made by at least two psychiatrists of the research team, and a consensus was reached via discussion where necessary. Healthy controls (HC) were excluded if they had a history of major Axis I psychiatric disorders, psychiatric illnesses in first-degree relatives, and/or childhood trauma. The exclusion criteria were as follows: 1) intellectual disability or any evidence of organic brain injury, 2) any physical conditions affecting mental states except treatable thyroid disorders, 3) difficulty understanding Korean, and 4) contraindications to MRI scanning. This study was performed according to the guidelines of the Helsinki Declaration, and approved by the Institutional Review Board of the National Center for Mental Health (NCMH IRB 116271-2018-04). Written informed consent was acquired from all participants after a complete study description was provided.

\section{Assessment of clinical variables}

Clinical characteristics of bipolar patients were acquired through the Korean version of the MINI. The severity of BD was evaluated based on the Clinical Global Impression for Bipolar Disorder-Overall Severity (CGI-BP-OS). ${ }^{26}$ The levels of childhood trauma, coexisting anxiety, and impulsivity in the patient group were assessed with standard self-reported scales; the Korean version-Childhood Trauma Questionnaire (CTQ), ${ }^{27}$ the Korean version-Beck Anxiety Inventory (BAI), ${ }^{28}$ and the Korean version-Barratt Impulsiveness Scale (BIS), ${ }^{29}$ respectively. The CTQ is a widely used screening tool which aims to detect experiences of childhood abuse and neglect in adults and adolescents. ${ }^{30}$ The reliability of the CTQ has been demonstrated in patients with $\mathrm{BD} .{ }^{31}$ In addition, we intended to measure the severity of current anxiety and impulsivity. The BAI is a reliable instrument for evaluating current anxiety. ${ }^{32}$ The BIS was used to assess the severity of current impulsivity, ${ }^{33}$ and the reliability of the BIS has been proved in patients with BD. ${ }^{34}$ All of these self-reported scales have been translated to Korean and validated in Korean subjects. ${ }^{35-37}$ 


\section{Image data acquisition}

Brain images of the patients and $\mathrm{HC}$ were obtained using 3 Tesla MRI scanner (Ingenia CX, Philips, Erlangen, Germany) equipped with a 32-channel head coil at the National Center for Mental Health. High-resolution anatomical T1-weighted (T1W) images were acquired with a turbo field echo sequence (spin-echo TR=9.8 ms, TE=4.6 ms, matrix size: $240 \times 240,170$ sagittal slices, $\mathrm{FOV}=240 \mathrm{~mm}$, slice thickness $=1 \mathrm{~mm}$, and flip angle $=8^{\circ}$ ).

\section{Image data processing and analyses}

T1W images of the subjects were processed through the VBM analysis pipeline of the Statistical Parametric Mappingversion 12 (SPM12) software (Wellcome Department of Imaging Neuroscience, University College, London, UK) (http:// www.fil.ion.ucl.ac.uk/spm/). The T1W images were segmented into gray matter, white matter, and other including cerebrospinal fluid, using the New Segmentation option in SPM12. Segmented T1W gray matter maps were warped to the Montreal Neurological Institute (MNI) 152 template. The goal of this normalization procedure was to reflect individual characteristics of cranial morphology and improve the accuracy of the analysis. Next, modulation was conducted by multiplying gray matter images to the Jacobian determinates of the deformation fields from the normalization process. The modulation ensured that the total amount of gray matter in each voxel was preserved. Finally, modulated gray matter images were smoothed with a Gaussian kernel of $8 \times 8 \times 8 \mathrm{~mm}^{3}$ full width at half-maximum (FWHM).

We initially carried out voxel-wise group comparisons of GMV over the whole brain between BD patients and HC using analysis of covariance (ANCOVA) with age, sex and intracranial volume (ICV) as covariates. Clusters were reported as significantly different if they survived the false discovery rate (FDR) correction for multiple comparisons $(\mathrm{p}<0.01)$.

Finally, partial correlation analyses were conducted within the $\mathrm{BD}$ group to examine the correlations between mean values of GMV in the regions that survived the group comparisons and the scores of the CTQ, BAI, and BIS, controlling for age, sex and ICV $(\mathrm{p}<0.05)$. To explore the effects of other clinical variables on the correlations, further partial correlation analyses were performed with additional covariates such as onset age, illness duration, number of mood episodes, and the CGI-BP-OS.

\section{Statistical analyses of clinical and socio-demographic data}

To compare demographic data between the $\mathrm{BD}$ group and $\mathrm{HC}$, independent-sample t-tests and chi-square tests were employed for continuous and categorical variables, respec- tively. A partial correlation analysis was performed to investigate the relationships between the clinical scales (CTQ, BAI, and BIS), adjusting for the CGI-BP-OS scores to minimize confounding effects of current mood states. Moreover, additional partial correlation analyses between the scales were conducted adjusting for onset age, illness duration, number of mood episodes and the CGI-BP-OS individually and concomitantly to investigate the effects of various clinical factors on the relationships.

All statistical analyses were conducted using Statistical Package for the Social Science (SPSS) (IBM Corp., Released 2012. IBM SPSS Statistics for Windows, Version 21.0. IBM Corp., Armonk, NY, USA).

\section{RESULTS}

\section{Demographics and clinical characteristics of subjects}

Demographic and clinical data of the participants are presented in Table 1 . The mean age of the $36 \mathrm{BD}$ patients was 30.6 years, and $55.6 \%$ of them were female. There were no significant differences in age and gender between the patients and the HC. The BD patients had significantly shorter dura-

Table 1. Demographic and clinical characteristics of the subjects

\begin{tabular}{lcc}
\hline & $\begin{array}{c}\text { Patients } \\
(\mathrm{N}=36)\end{array}$ & $\begin{array}{c}\text { Controls } \\
(\mathrm{N}=29)\end{array}$ \\
\hline Demographics & & \\
Age (years, mean $\pm \mathrm{SD})$ & $30.6 \pm 8.3$ & $29.3 \pm 5.3$ \\
Female (\%) & 55.6 & 65.6 \\
Duration of education (year, mean $\pm \mathrm{SD})$ & $13.3 \pm 2.1^{*}$ & $16.1 \pm 1.0$ \\
Other clinical characteristics & & \\
BD type I (\%) & 88.9 & N/A \\
Onset age (year, mean $\pm \mathrm{SD})$ & $22.7 \pm 6.0$ & N/A \\
Illness duration (year, mean $\pm \mathrm{SD})$ & $7.9 \pm 8.3$ & N/A \\
Number of mood episodes (mean $\pm \mathrm{SD})$ & $6.6 \pm 8.0$ & N/A \\
CGI-BP-OS (current, mean $\pm \mathrm{SD})$ & $5.0 \pm 1.3$ & N/A \\
CTQ (mean $\pm S D)$ & $50.8 \pm 15.5$ & N/A \\
BAI (mean $\pm S D)$ & $13.2 \pm 13.1$ & N/A \\
BIS (mean $\pm S D)$ & $59.9 \pm 14.4$ & N/A \\
Current psychotropic use & & \\
Number of psychotropics (mean $\pm S D)$ & $2.8 \pm 0.7$ & N/A \\
Complex pharmacotherapy (\%) & 100.0 & N/A \\
Antipsychotics (\%) & 88.9 & N/A \\
Mood stabilizers (\%) & 88.9 & N/A \\
\hline
\end{tabular}

${ }^{*} \mathrm{p}<0.05$ bipolar patients versus healthy controls. SD: standard deviation, BD: bipolar disorder, CGI-BP-OS: Clinical Global Impression for Bipolar Disorder-Overall Severity, CTQ: Childhood Trauma Questionnaire, BAI: Beck Anxiety Inventory, BIS: Barratt Impulsiveness Scale 
tions of education than HC. The majority of the patients were diagnosed with BD I. Mean onset age was 22.7 years and mean illness duration was 7.9 years, with 6.6 mood episodes on average. The mean CGI-BP-OS scores of the patients was 5.0, and the mean CTQ, BAI, and BIS scores were 50.8, 13.2, and 59.9, respectively. All patients were receiving combination pharmacotherapy with 2.8 psychotropic medications on average, at least one antipsychotics or mood stabilizer.

\section{Relationships between childhood trauma, anxiety, and impulsivity}

The partial correlation analysis adjusted for the CGI-BPOS scores revealed significantly positive correlations between the CTQ, BAI, and BIS scores (Table 2). The CTQ showed significantly positive associations with the BAI ( $r=0.439$, $\mathrm{p}=0.008)$ and the BIS $(\mathrm{r}=0.488, \mathrm{p}=0.003)$. In addition, there was a significantly positive association between the BAI and $\mathrm{BIS}(\mathrm{r}=0.480, \mathrm{p}=0.004)$. These correlations remained significantly after adjusting for the CGI-BP-OS, onset age, illness duration, and number of mood episodes individually and concomitantly. These findings suggest that the severity of childhood trauma, current anxiety, and impulsivity in $\mathrm{BD}$ are significantly interrelated.

Table 2. Partial correlation analysis between CTQ, BAI, and BIS scores in BD group

\begin{tabular}{llll}
\hline & CTQ & BAI & BIS \\
\hline CTQ & 1 & & \\
BAI & $0.439^{*}$ & 1 & \\
BIS & $0.488^{*}$ & $0.480^{*}$ & 1 \\
\hline
\end{tabular}

${ }^{*} \mathrm{p}<0.01$. CTQ: Childhood Trauma Questionnaire, BAI: Beck Anxiety Inventory, BIS: Barratt Impulsiveness Scale, BD: bipolar disorder

\section{Group comparisons of GMV between BD patients and $\mathrm{HC}$}

Patients with BD compared to HC showed significantly decreased GMV in multiple cortical and subcortical areas including the bilateral hippocampus, bilateral precentral gyrus, left inferior temporal gyrus, right fusiform gyrus, left thalamus, right insular lobe, and left middle frontal gyrus (Table 3). According to antecedent literatures, the temporal cortex involving the hippocampal-amygdala complex, the thalamus, and the middle frontal gyrus within the prefrontal area seem to be the main brain structures involved in the pathophysiology of $\mathrm{BD} \cdot{ }^{38-40}$ No brain region exhibited significantly increased GMV in the BD group compared to HC.

\section{Associations between regional GMV alterations and clinical variables in $\mathrm{BD}$ patients}

The results of the VBM analyses are presented in Figure 1. Patients with BD showed a significant inverse correlation between the mean GMV of the right precentral gyrus and the CTQ score $(r=-0.609, p<0.0003)$, and a similar tendency of a negative association between the GMV of the right precentral gyrus and the BIS score $(\mathrm{r}=-0.336, \mathrm{p}=0.065)$. Moreover, there was a significant inverse correlation between the mean GMV of the left middle frontal gyrus and the BAI score $(\mathrm{r}=-0.363$, $\mathrm{p}=0.044$ ), and a similar tendency of a negative association between the GMV of the left middle frontal gyrus and the CTQ score $(\mathrm{r}=-0.339, \mathrm{p}=0.062)$.

With respect to the right precentral gyrus, these correlations remained significantly after adjusting for onset age, illness duration, number of mood episodes, and the CGI-BP-OS. For the left middle frontal gyrus, the findings still appeared with adjusting for number of mood episodes and CGI-BP-OS whereas the statistical power was moderately weakened when adjust-

Table 3. Brain regions with significant differences in GMV between BD patients and HC

\begin{tabular}{|c|c|c|c|c|c|c|c|}
\hline \multirow{2}{*}{ Anatomical region } & \multirow{2}{*}{ Side } & \multirow{2}{*}{$\mathrm{t} \max$} & \multicolumn{3}{|c|}{ Peak coordinates (MNI) } & \multirow{2}{*}{ Cluster size (voxels) } & \multirow{2}{*}{$\mathrm{p}$ value (FDR corrected) } \\
\hline & & & $\mathrm{x}$ & $\mathrm{y}$ & $\mathrm{z}$ & & \\
\hline \multicolumn{8}{|l|}{$\mathrm{BD}<\mathrm{HCs}$} \\
\hline Hippocampus & $\mathrm{L}$ & 6.135 & -36 & -4.5 & -22 & 4,843 & 0.0024 \\
\hline Precentral gyrus & $\mathrm{L}$ & 5.468 & -45 & -4.5 & 28 & 685 & 0.0024 \\
\hline Hippocampus & $\mathrm{R}$ & 4.858 & 28.5 & -9 & -16 & 392 & 0.0041 \\
\hline Precentral gyrus & $\mathrm{R}$ & 4.633 & 34.5 & -16.5 & 39 & 297 & 0.0058 \\
\hline Inferior temporal gyrus & $\mathrm{L}$ & 4.878 & -57 & -24 & -24 & 213 & 0.0040 \\
\hline Fusiform gyrus & $\mathrm{R}$ & 4.650 & 21 & -48 & -15 & 159 & 0.0057 \\
\hline Thalamus & $\mathrm{L}$ & 4.524 & 1.5 & -7.5 & 9 & 115 & 0.0069 \\
\hline Insular lobe & $\mathrm{R}$ & 4.480 & 33 & 28.5 & -1 & 98 & 0.0073 \\
\hline Middle frontal gyrus & $\mathrm{L}$ & 4.626 & -18 & 46.5 & 15 & 81 & 0.0059 \\
\hline
\end{tabular}

Note that all MNI coordinates of maximum t values are selected in the significant regions. GMV: gray matter volume, BD: bipolar disorder, HC: healthy controls, MNI: Montreal Neurological Institute, FDR: false discovery rate, L: left, R: right 


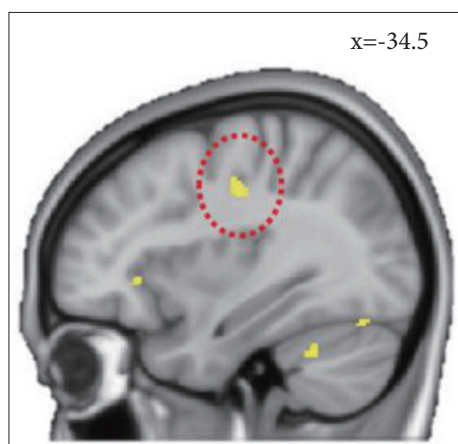

A

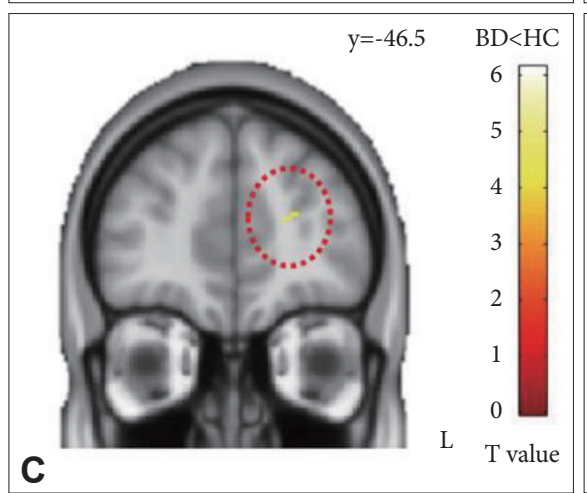

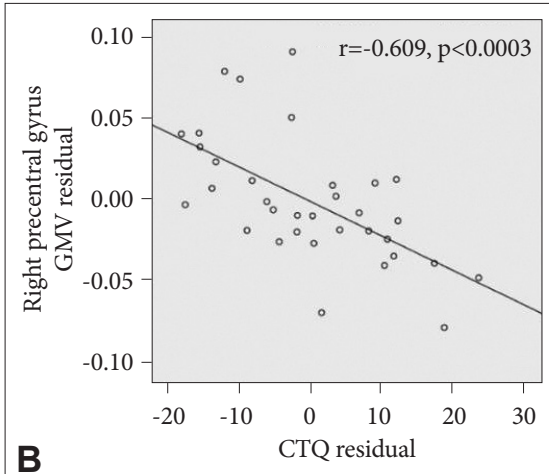
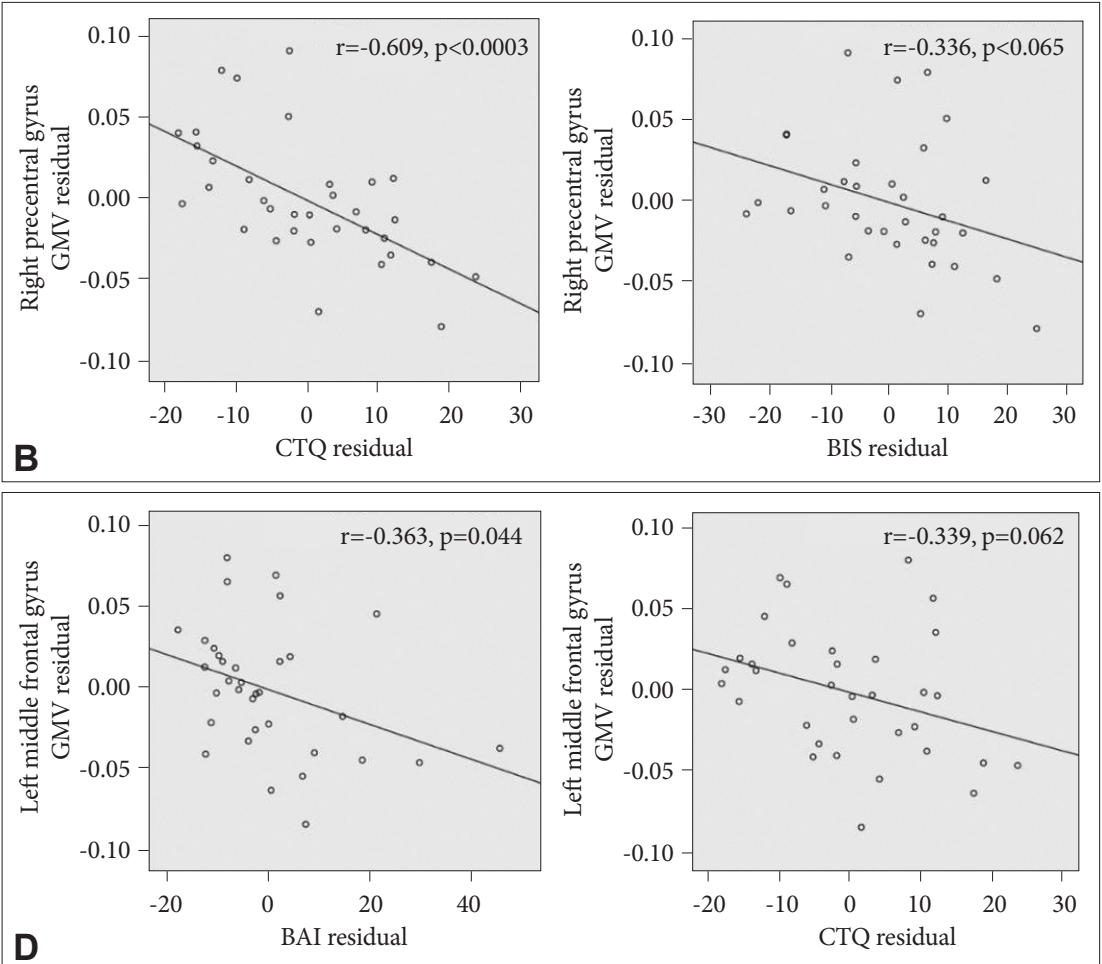

Figure 1. Brain regions of correlations with childhood trauma, current anxiety, and impulsivity in the bipolar group. Red circles indicate (A) the right precentral gyrus and (C) the left middle frontal gyrus. (B) Partial correlations between the clinical scale scores (CTQ and BIS) and GMV in the right precentral gyrus. (D) Partial correlations between the clinical scale scores (BAI and CTQ) and GMV in the left middle frontal gyrus. Linear regression fit lines were created using the least squares method. To determine partial correlations, variables were regressed onto covariates using a linear regression. Calculated non-standardized residuals were used to produce scatter plots. BD: bipolar disorder, HC: healthy controls, GMV: gray matter volume, CTQ: Childhood Trauma Questionnaire, BIS: Barratt Impulsiveness Scale, BAI: Beck Anxiety Inventory.

ing for onset age or illness duration. The tendency of the inverse relationships between the mean GMV of the left middle frontal gyrus and the score of above-mentioned scales was still maintained.

\section{DISCUSSION}

The main results of this study are as follows: 1) the severity of childhood trauma, comorbid anxiety, and impulsivity were interrelated in patients with $\mathrm{BD}, 2) \mathrm{BD}$ patients showed significant inverse correlations between the GMV in the right precentral gyrus and CTQ scores; between the GMV in the left middle frontal gyrus and BAI scores, and 3) BD patients exhibited similar tendencies of negative associations between the GMV in the right precentral gyrus and BIS scores; between the GMV in the left middle frontal gyrus and CTQ scores. Our findings support the hypothesis that childhood trauma, anxiety, and impulsivity are correlated with GMV alterations in overlapping regions within the $\mathrm{BD}$ group.

There is an extensive body of neuroimaging literatures which elucidate the neurobiological mechanisms underlying $\mathrm{BD}$. However, the mainstream methodology in previous stud- ies has been a group comparison between patients and normal individuals, or between $\mathrm{BD}$ patients with and without a certain clinical factor. It is a strength of our study that we explored correlations among various factors emerging as indicators of worse outcomes of $\mathrm{BD}$ and investigated the relationships between these clinical variables and neuroanatomical changes within a bipolar group.

In the recent years interest has been paid to the relationships among childhood trauma, current anxiety, and impulsivity, but the evidence for such links in $\mathrm{BD}$ is limited. Our findings are in agreement with the few preceding studies, which reported that childhood abuse was significantly associated with affective instability in $\mathrm{BD}$ regardless of current mood states ${ }^{17}$ and that subjects with $\mathrm{BD}$ showed considerably higher impulsivity when anxiety was present either as a comorbidity or a symptom. ${ }^{18}$ In bipolar patients, adverse experiences in early life may affect mood dysregulation based on a neurobiological mechanism linked to anxiety and impulsivity. ${ }^{17,41}$

In accordance with the significant associations between childhood trauma, anxiety, and impulsivity, our neuroimaging study also indicated that the cortical volumetric correlates of the three clinical variables overlapped in the right precentral 
gyrus and the left middle frontal gyrus within the BD group. Previous studies pointed out that decreased GMV of the orbitofrontal cortex in $\mathrm{BD}$ patients compared to a healthy group was only observed in subjects with a history of childhood maltreatment, ${ }^{22}$ and that CTQ scores in a BD group were inversely correlated with GMV in the right dorsolateral prefrontal cortex and the right thalamus. ${ }^{19}$ We found a significantly negative correlation between the CTQ scores and the GMV in the right precentral gyrus, and a similar tendency in the left middle frontal gyrus. There results may represent neural evidence of the impact of childhood abuse on emotional control in BD. In addition, a few neuroimaging studies reported that bipolar patients with anxiety and impulsivity exhibited GMV loss in the posteromedial rectal gyrus and the anterior cingulate cortex, respectively. ${ }^{42,43}$ We observed significant associations between GMV deficits in the left middle frontal gyrus and anxiety, and a similar tendency between GMV deficits in the right precentral gyrus and impulsivity. Although these correlations in the left middle frontal gyrus might be affected by onset age or illness duration, similar tendency of the findings remained in the present study. Taken together, our results on cortical volumetric correlates of childhood trauma are partially replicated, whereas neuronal correlates of anxiety and impulsivity are inconsistent with earlier researches. This discrepancy may be due to heterogeneity of subject samples as well as dissimilarities in methods including image acquisition, processing and analysis.

The precentral gyrus, identified as a structural correlate of childhood trauma in $\mathrm{BD}$ in this study, includes the primary motor cortex responsible for voluntary movement. It is also believed that the precentral gyrus is involved in cognitive processing and emotional regulation. ${ }^{44-46}$ Several structural MRI studies demonstrated abnormalities in the precentral gyrus in $\mathrm{BD}$, and provided evidence that the dysfunction in the bipolar brain occurred not only in regions related to emotional processing, but also in the traditional motor cortex-precentral gyrus. ${ }^{47-49}$ The middle frontal gyrus, discovered as a structural correlate of current anxiety in BD in the present study, is located in the prefrontal areas and plays an important role in the prefrontal-limbic system. The middle frontal gyrus includes parts of Brodmann areas (BA) 9, 10, and 46, which are related to the emotion regulation and pathophysiology of $\mathrm{BD}^{50-54} \mathrm{BA} 9$ on the left has been reported to be associated with the processing of positive and negative emotional information. ${ }^{55,56} \mathrm{BA} 10$, one of the most poorly understood regions in the human brain, is possibly engaged in integrating consequences of multiple cognitive operations for achieving a higher goal in the next behavior. ${ }^{54}$ Furthermore, BA 46 is thought to be involved in high-level cognitive processing such as planning. ${ }^{57}$ Earlier studies have revealed that the middle frontal gyrus has dense connections with the amygdala and other limbic structures related to emotional regulation. ${ }^{58}$ Meanwhile, a meta-study reported that associations between cortical thickness in $\mathrm{BD}$ and illness duration or onset age were mostly non-significant and there was the scarcity of study pointing out that cortical thinning was related to illness duration of $\mathrm{BD}$ in certain frontal areas such as left middle frontal cortex. ${ }^{59,60}$ In summary, the current findings present evidence of abnormalities in multiple frontal lobe areas in $\mathrm{BD}$ that process nonverbal cues as well as emotional stimuli associated with childhood trauma and affect regulation.

This study has some clinical and academic implications. First, histories of childhood trauma, comorbid anxiety, and impulsivity need to be simultaneously considered and appropriately assessed in the management of $\mathrm{BD}$. Literature review has emphasized these clinical factors as determinants of unfavorable outcomes of $\mathrm{BD}$ and risk factors for suicidality. ${ }^{11,13,61,62}$ One previous study in particular proposed that bipolar populations with early life stress and comorbid anxiety might comprise a distinct etiological type of $\mathrm{BD} .{ }^{61}$ Our findings suggest that bipolar patients with a history of childhood trauma, current anxiety, and impulsivity may constitute a discrete group with a worse prognosis. Second, the GMV alterations in the precentral gyrus and middle frontal gyrus may be used as potential neurobiological markers for the categorization of patients with $\mathrm{BD}$, or as a complementary tool for diagnosing and staging $\mathrm{BD}{ }^{63,64}$ These implications can contribute to a more individualized assessment and treatment of BD.

We have noticed some limitations in this study. First, the relatively small sample size and the enrollment from only one institution may limit the generalizability of the present findings. The number of subjects in recent studies investigating neuronal correlates of clinical variables in mood disorders was $20-39 .^{19,20,65}$ However, we recruited not only patients but also $\mathrm{HC}$ to overcome this limitation. Second, effects of psychotropic medications on brain structures may need to be considered. Nonetheless, the statistical effect of such treatment on the present findings seemed to be very weak, since our patient sample was very homogenous regarding psychotropic medications (Table 1). ${ }^{66}$ Third, the scales measuring clinical variables (CTQ, BAI, and BIS) were all self-reported. Especially for the CTQ, a retrospective report, biases may occur when adults recall adverse memories from their childhood. ${ }^{67}$ Nevertheless, a longitudinal cohort study reported that CTQ scores were consistent with prospective self-reports of childhood trauma exposure ${ }^{68}$ Fourth, the design of this study was cross-sectional, based on an one-time assessment. Longitudinal investigations may be helpful to underpin the causal relationships among childhood trauma, structural brain changes, and clinical outcomes of $\mathrm{BD}^{69}$

Despite these limitations, this is the first study demonstrat- 
ing that in $\mathrm{BD}$, childhood trauma, anxiety, and impulsivity are interrelated and that the cortical volumetric correlates of these clinical variables overlap in the right precentral gyrus and the left middle frontal gyrus. The present findings suggest that childhood trauma, anxiety, and impulsivity may affect outcomes of BD based on neural mechanism, and that the GMV changes could be used as neurobiological markers predicting the prognosis of BD. Further studies are warranted to establish more evidence for the application of the current results in clinical practice.

\section{Acknowledgments}

This research was supported by a grant from the National Center for Mental Health (2019-01). The current data were also presented at the regional meeting of the Korean Neuropsychiatric Association in Busan, October 12-13, 2018.

\section{Conflicts of Interest}

The authors have no potential conflicts of interest to disclose.

\section{Author Contributions}

Conceptulization: Vin Ryu, Dong Yeon Park. Data curation: Hyehyun Song, Rina Yu. Formal analysis: Hyeongrae Lee, Wonhye Lee. Funding acquisition: Dong Yeon Park. Investigation: Hyehyun Song, Rina Yu, Dong Yeon Park. Methodology: Dong-Kyun Lee. Project administration: Dong Yeon Park. Resources: Vin Ryu, Rina Yu, Jung Hyun Lee. Software: DongKyun Lee. Supervision: Myong-Wuk Chon, Hyeongrae Lee, Dong Yeon Park. Validation: Myong-Wuk Chon, Hyeongrae Lee, Wonhye Lee, Dong Yeon Park. Visualization: Hyeongrae Lee, Wonhye Lee. Writing_original draft: Hyehyun Song, Dong Yeon Park. Writing_review \& editing: Dong Yeon Park.

\section{ORCID iDs}

\section{Hyehyun Song}

Myong-Wuk Chon

Vin Ryu

Rina Yu

Dong-Kyun Lee

Hyeongrae Lee

Wonhye Lee

Jung Hyun Lee

Dong Yeon Park https://orcid.org/0000-0003-3021-0634 https://orcid.org/0000-0002-1800-1517 https://orcid.org/0000-0003-4840-7400 https://orcid.org/0000-0003-3107-4179 https://orcid.org/0000-0001-7191-000X https://orcid.org/0000-0002-5146-680X https://orcid.org/0000-0001-5464-3069 https://orcid.org/0000-0001-9116-4593 https://orcid.org/0000-0002-6972-722X

\section{REFERENCES}

1. Grande I, Berk M, Birmaher B, Vieta E. Bipolar disorder. Lancet 2016; 387:1561-1572.

2. Park DY, Goffin KC, Shah S, Yuen LD, Holtzman JN, Hooshmand F, et al. Differential prevalence and demographic and clinical correlates of second-generation antipsychotic use in bipolar I versus bipolar II disorder. J Psychiatr Res 2016;76:52-58.

3. Passos IC, Mwangi B, Vieta E, Berk M, Kapczinski F. Areas of controversy in neuroprogression in bipolar disorder. Acta Psychiatr Scand 2016;134:91-103.

4. Reinares M, Papachristou E, Harvey P, Mar Bonnin C, Sanchez-Moreno J, Torrent C, et al. Towards a clinical staging for bipolar disorder: defining patient subtypes based on functional outcome. J Affect Disord 2013;144:65-71.

5. Schaffer A, Isometsa ET, Tondo L, Moreno DH, Turecki G, Reis C, et al. International Society for Bipolar Disorders Task Force on Suicide: meta-analyses and meta-regression of correlates of suicide attempts and suicide deaths in bipolar disorder. Bipolar Disord 2015;17:1-16,

6. Sugaya L, Hasin DS, Olfson M, Lin KH, Grant BF, Blanco C. Child physical abuse and adult mental health: a national study. J Trauma Stress 2012;25:384-392.

7. Agnew-Blais J, Danese A. Childhood maltreatment and unfavourable clinical outcomes in bipolar disorder: a systematic review and metaanalysis. Lancet Psychiatry 2016;3:342-349.

8. Post RM, Altshuler LL, Kupka R, McElroy SL, Frye MA, Rowe M, et al. Verbal abuse, like physical and sexual abuse, in childhood is associated with an earlier onset and more difficult course of bipolar disorder. Bipolar Disord 2015;17:323-330.

9. Azorin JM, Kaladjian A, Adida M, Hantouche EG, Hameg A, Lancrenon S, et al. Psychopathological correlates of lifetime anxiety comorbidity in bipolar I patients: findings from a French national cohort. Psychopathology 2009;42:380-386.

10. Pavlova B, Perlis RH, Alda M, Uher R. Lifetime prevalence of anxiety disorders in people with bipolar disorder: a systematic review and meta-analysis. Lancet Psychiatry 2015;2:710-717.

11. Pavlova B, Perroud N, Cordera P, Uher R, Alda M, Dayer A, et al. Anxiety disorders and childhood maltreatment as predictors of outcome in bipolar disorder. J Affect Disord 2018;225:337-341.

12. Perroud N, Baud P, Preisig M, Etain B, Bellivier F, Favre S, et al. Social phobia is associated with suicide attempt history in bipolar inpatients. Bipolar Disord 2007;9:713-721.

13. Johnson SL, Carver CS, Tharp JA. Suicidality in bipolar disorder: the role of emotion-triggered impulsivity. Suicide Life Threat Behav 2017; 47:177-192.

14. Malloy-Diniz LF, Neves FS, de Moraes PH, De Marco LA, RomanoSilva MA, Krebs MO, et al. The 5-HTTLPR polymorphism, impulsivity and suicide behavior in euthymic bipolar patients. J Affect Disord 2011;133:221-226.

15. Swann AC, Dougherty DM, Pazzaglia PJ, Pham M, Steinberg JL, Moeller FG. Increased impulsivity associated with severity of suicide attempt history in patients with bipolar disorder. Am J Psychiatry 2005;162:1680-1687.

16. Jimenez E, Arias B, Mitjans M, Goikolea JM, Ruiz V, Brat M, et al. Clinical features, impulsivity, temperament and functioning and their role in suicidality in patients with bipolar disorder. Acta Psychiatr Scand 2016;133:266-276.

17. Marwaha S, Gordon-Smith K, Broome M, Briley PM, Perry A, Forty L, et al. Affective instability, childhood trauma and major affective disorders. J Affect Disord 2016;190:764-771.

18. Bellani M, Hatch JP, Nicoletti MA, Ertola AE, Zunta-Soares G, Swann $\mathrm{AC}$, et al. Does anxiety increase impulsivity in patients with bipolar disorder or major depressive disorder? J Psychiatr Res 2012;46:616-621.

19. Duarte DG, Neves Mde C, Albuquerque MR, de Souza-Duran FL, Busatto G, Correa H. Gray matter brain volumes in childhood-maltreated patients with bipolar disorder type I: a voxel-based morphometric study. J Affect Disord 2016;197:74-80.

20. Simeonova DI, Jackson V, Attalla A, Karchemskiy A, Howe M, Adleman N, et al. Subcortical volumetric correlates of anxiety in familial pediatric bipolar disorder: a preliminary investigation. Psychiatry Res 2009;173:113-120.

21. Strakowski SM, DelBello MP, Adler C, Cecil KM, Sax KW. Neuroimaging in bipolar disorder. Bipolar Disord 2000;2:148-164.

22. Poletti S, Vai B, Smeraldi E, Cavallaro R, Colombo C, Benedetti F. Adverse childhood experiences influence the detrimental effect of bipolar disorder and schizophrenia on cortico-limbic grey matter volumes. J Affect Disord 2016;189:290-297.

23. Sheehan DV, Lecrubier Y, Sheehan KH, Amorim P, Janavs J, Weiller E, et al. The Mini-International Neuropsychiatric Interview (M.I.N.I): the development and validation of a structured diagnostic psychiatric interview for DSM-IV and ICD-10. J Clin Psychiatry 1998;59:22-33.

24. Yoo SW, Kim YS, Noh JS, Oh KS, Kim CH, NamKoong K, et al. Validity of Korean version of the mini-international neuropsychiatric inter- 
view. Anxiety Mood 2006;2:50-55.

25. American Psychiatric Association. Diagnostic and Statistical Manual of Mental Disorders (DSM- $5^{\circledR}$ ). Arlington, VA: American Psychiatric Pub; 2013.

26. Spearing MK, Post RM, Leverich GS, Brandt D, Nolen W. Modification of the Clinical Global Impressions (CGI) Scale for use in bipolar illness (BP): the CGI-BP. Psychiatry Res 1997;73:159-171.

27. Bernstein DP, Stein JA, Newcomb MD, Walker E, Pogge D, Ahluvalia T, et al. Development and validation of a brief screening version of the Childhood Trauma Questionnaire. Child Abuse Negl 2003;27:169-190.

28. Beck AT, Epstein N, Brown G, Steer RA. An inventory for measuring clinical anxiety: psychometric properties. J Consult Clin Psychol 1988; 56:893-897.

29. Patton JH, Stanford MS, Barratt ES. Factor structure of the Barratt impulsiveness scale. J Clin Psychol 1995;51:768-774.

30. Grassi-Oliveira R, Cogo-Moreira H, Salum GA, Brietzke E, Viola TW, Manfro GG, et al. Childhood Trauma Questionnaire (CTQ) in Brazilian samples of different age groups: findings from confirmatory factor analysis. PLoS One 2014;9:e87118.

31. Etain B, Mathieu F, Henry C, Raust A, Roy I, Germain A, et al. Preferential association between childhood emotional abuse and bipolar disorder. J Trauma Stress 2010;23:376-383.

32. Fydrich T, Dowdall D, Chambless DL. Reliability and validity of the Beck Anxiety Inventory. J Anxiety Disord 1992;6:55-61.

33. Stanford MS, Mathias CW, Dougherty DM, Lake SL, Anderson NE, Patton JH. Fifty years of the Barratt Impulsiveness Scale: an update and review. Pers Individ Diff 2009;47:385-395.

34. Peluso M, Hatch J, Glahn D, Monkul E, Sanches M, Najt P, et al. Trait impulsivity in patients with mood disorders. J Affect Disord 2007; 100:227-231.

35. Kim D, Park SC, Yang H, Oh DH. Reliability and validity of the Korean version of the childhood trauma questionnaire-short form for psychiatric outpatients. Psychiatry Investig 2011;8:305-311.

36. Yook S, Kim Z. A clinical study on the Korean version of Beck Anxiety Inventory: comparative study of patient and non-patient. Korean J Clin Psychol 1997;16:185-197.

37. Lee SR, Lee WH, Park JS, Kim SM, Kim JW, Shim JH. The study on Reliability and Validity of Korean Version of the Barratt Impulsiveness Scale-11-Revised in nonclinical adult subjects. J Korean Neuropsychiatr Assoc 2012;51:378-386.

38. Price JL, Drevets WC. Neurocircuitry of mood disorders. Neuropsychopharmacology 2010;35:192-216.

39. Maletic V, Raison C. Integrated neurobiology of bipolar disorder. Front Psychiatry 2014;5:98.

40. Sheline YI. Neuroimaging studies of mood disorder effects on the brain. Biol Psychiatry 2003;54:338-352.

41. Aas M, Henry C, Andreassen OA, Bellivier F, Melle I, Etain B. The role of childhood trauma in bipolar disorders. Int J Bipolar Disord 2016;4:2.

42. Almeida JR, Akkal D, Hassel S, Travis MJ, Banihashemi L, Kerr N, et al. Reduced gray matter volume in ventral prefrontal cortex but not amygdala in bipolar disorder: significant effects of gender and trait anxiety. Psychiatry Res 2009;171:54-68.

43. Matsuo K, Nicoletti MA, Peluso MA, Hatch JP, Nemoto K, Watanabe $\mathrm{Y}$, et al. Anterior cingulate volumes associated with trait impulsivity in individuals with bipolar disorder. Bipolar Disord 2009;11:628-636.

44. Seo D, Olman CA, Haut KM, Sinha R, MacDonald AW 3rd, Patrick CJ. Neural correlates of preparatory and regulatory control over positive and negative emotion. Soc Cogn Affect Neurosci 2014;9:494-504.

45. Thomas LA, Brotman MA, Bones BL, Chen G, Rosen BH, Pine DS, et al. Neural circuitry of masked emotional face processing in youth with bipolar disorder, severe mood dysregulation, and healthy volunteers. Dev Cogn Neurosci 2014;8:110-120.

46. Zhao L, Wang Y, Jia Y, Zhong S, Sun Y, Qi Z, et al. Altered interhemispheric functional connectivity in remitted bipolar disorder: a resting state fMRI study. Sci Rep 2017;7:4698.
47. Eker C, Simsek F, Yilmazer EE, Kitis O, Cinar C, Eker OD, et al. Brain regions associated with risk and resistance for bipolar I disorder: a voxel-based MRI study of patients with bipolar disorder and their healthy siblings. Bipolar Disord 2014;16:249-261.

48. Fung G, Deng Y, Zhao Q, Li Z, Qu M, Li K, et al. Distinguishing bipolar and major depressive disorders by brain structural morphometry: a pilot study. BMC Psychiatry 2015;15:298.

49. Jorgensen KN, Nerland S, Norbom LB, Doan NT, Nesvag R, MorchJohnsen L, et al. Increased MRI-based cortical grey/white-matter contrast in sensory and motor regions in schizophrenia and bipolar disorder. Psychol Med 2016;46:1971-1985.

50. Goldin PR, McRae K, Ramel W, Gross JJ. The neural bases of emotion regulation: reappraisal and suppression of negative emotion. Biol Psychiatry 2008;63:577-586.

51. Rajkowska G, Goldman-Rakic PS. Cytoarchitectonic Definition of Prefrontal Areas in the Normal Human Cortex: I. Remapping of Areas 9 and 46 using Quantitative Criteria. Cereb Cortex 1995;5:307-322.

52. Rajkowska G, Goldman-Rakic PS. Cytoarchitectonic Definition of Prefrontal Areas in the Normal Human Cortex: II. Variability in Locations of Areas 9 and 46 and Relationship to the Talairach Coordinate System. Cereb Cortex 1995;5:323-337.

53. Phillips ML, Ladouceur CD, Drevets WC. A neural model of voluntary and automatic emotion regulation: implications for understanding the pathophysiology and neurodevelopment of bipolar disorder. Mol Psychiatry 2008;13:829, 833-857.

54. Ramnani N, Owen AM. Anterior prefrontal cortex: insights into function from anatomy and neuroimaging. Nat Rev Neurosci 2004;5:184-194.

55. Kerestes R, Ladouceur CD, Meda S, Nathan PJ, Blumberg HP, Maloney $\mathrm{K}$, et al. Abnormal prefrontal activity subserving attentional control of emotion in remitted depressed patients during a working memory task with emotional distracters. Psychol Med 2012;42:29-40.

56. Lane RD, Reiman EM, Bradley MM, Lang PJ, Ahern GL, Davidson RJ, et al. Neuroanatomical correlates of pleasant and unpleasant emotion. Neuropsychologia 1997;35:1437-1444.

57. Petrides M, Pandya DN. Dorsolateral prefrontal cortex: comparative cytoarchitectonic analysis in the human and the macaque brain and corticocortical connection patterns. Eur J Neurosci 1999;11:1011-1036.

58. Öngür D, Price J. The organization of networks within the orbital and medial prefrontal cortex of rats, monkeys and humans. Cereb Cortex 2000;10:206-219.

59. Hanford LC, Nazarov A, Hall GB, Sassi RB. Cortical thickness in bipolar disorder: a systematic review. Bipolar Disord 2016;18:4-18.

60. Lyoo IK, Sung YH, Dager SR, Friedman SD, Lee JY, Kim SJ, et al. Regional cerebral cortical thinning in bipolar disorder. Bipolar Disord 2006;8:65-74.

61. Pavlova B, Perroud N, Cordera P, Uher R, Dayer A, Aubry JM. Childhood maltreatment and comorbid anxiety in people with bipolar disorder. J Affect Disord 2016;192:22-27.

62. Richard-Lepouriel H, Kung AL, Hasler R, Bellivier F, Prada P, Gard S, et al. Impulsivity and its association with childhood trauma experiences across bipolar disorder, attention deficit hyperactivity disorder and borderline personality disorder. J Affect Disord 2019;244:33-41.

63. Kapczinski F, Dias VV, Kauer-Sant'Anna M, Brietzke E, Vazquez GH, Vieta E, et al. The potential use of biomarkers as an adjunctive tool for staging bipolar disorder. Prog Neuropsychopharmacol Biol Psychiatry 2009;33:1366-1371.

64. Phillips ML, Kupfer DJ. Bipolar disorder diagnosis: challenges and future directions. Lancet 2013;381:1663-1671.

65. Lee YJ, Kim S, Gwak AR, Kim SJ, Kang SG, Na KS, et al. Decreased regional gray matter volume in suicide attempters compared to suicide non-attempters with major depressive disorders. Compr Psychiatry 2016; 67:59-65.

66. Lyoo IK, Dager SR, Kim JE, Yoon SJ, Friedman SD, Dunner DL, et al. Lithium-induced gray matter volume increase as a neural correlate of treatment response in bipolar disorder: a longitudinal brain imaging 
study. Neuropsychopharmacology 2010;35:1743-1750.

67. Maughan B, Rutter M. Retrospective reporting of childhood adversity: issues in assessing long-term recall. J Pers Disord 1997;11:19-33.

68. Liebschutz JM, Buchanan-Howland K, Chen CA, Frank DA, Richardson MA, Heeren TC, et al. Childhood Trauma Questionnaire (CTQ) correlations with prospective violence assessment in a longitudinal cohort. Psychol Assess 2018;30:841-845.

69. Dusi N, De Carlo V, Delvecchio G, Bellani M, Soares JC, Brambilla P.
MRI features of clinical outcome in bipolar disorder: a selected review: special section on "Translational and neuroscience studies in affective disorders." Section Editor, Maria Nobile MD, PhD. This section of JAD focuses on the relevance of translational and neuroscience studies in providing a better understanding of the neural basis of affective disorders. The main aim is to briefly summaries relevant research findings in clinical neuroscience with particular regards to specific innovative topics in mood and anxiety disorders. J Affect Disord 2019;243:559-563. 